\title{
El papel de la negociación en la planeación de la estrategia militar
}

| Fecha de recibido: 2 de junio del 2021 | Fecha de aprobación: 27 de septiembre del 2021 |

Carlos Alberto

Segura Villarreal

Máster en Gerencia

y Negociaciones Internacionales

KUMIAY Internacional Co. Ltd

Japón

Rol del investigador: teórico https://orcid.org/0000-0002-6730-5584

$\bowtie$ csegura@kumiay.com

Cómo citar este artículo: Segura-Villarreal, C. (2021). El papel de la negociación en la planeación de la estrategia militar. Ciencia y Poder Aéreo, 16(2), 82-97. https://doi.org/10.18667/cienciaypoderaereo.735 


\section{El papel de la negociación en la planeación de la estrategia militar}

\section{The Role of Negotiation in Planning a Military Strategy}

\section{O papel da negociação no planejamento da estratégia militar}

Resumen: Para la planificación de una estrategia efectiva, eficaz y capaz de arrojar los resultados deseados, es necesario que todas las partes que interactúan con el proceso estratégico militar estén conscientes de la importancia de la negociación y de cómo esta sirve de herramienta para llegar a la planeación estratégica deseada. Con esta investigación no experimental de tipo descriptiva con enfoque cualitativo y diseño transversal, se busca dar respuesta la pregunta: ¿cómo la negociación puede contribuir en la planeación de la estrategia militar? Para ello, primero se analizan los conceptos básicos de estrategia, innovación y negociación. Como segundo punto, se identifica el enfoque primordial o método directo en una negociación exitosa y, en tercer lugar, se expone de qué forma contribuyen las negociaciones en el desarrollo estratégico. Al terminar de leer este artículo, el lector podrá comprender mejor cómo la negociación contribuye a la planeación estratégica militar, también, el cómo poder hacer uso de herramientas básicas para la planeación de una negociación, lo cual le traerá beneficios en su planeación estratégica.

Palabras clave: control estratégico; estrategia; innovación; negociación; planeación.

Abstract: Planning an effective and efficient strategy capable of producing the expected results demands that the parties involved in the military strategic process acknowledge the importance of negotiation, and also serves as a tool to obtain the desired strategic planning. This non-experimental and descriptive research, under a qualitative focus and a cross-sectional design, seeks to answer how negotiation could contribute to the planning of a military strategy. To address this question, we will examine the basic concepts of strategy, innovation, and negotiation. In addition, the main approach or direct method in a successful negotiation will be identified. Moreover, we will expose how negotiation contributes to strategic development. Upon finishing this article, the reader will be able to comprehend how a negotiation contributes to the military strategic planning process and how to deploy basic planning tools for a negotiation, which will bring benefits to their own strategic planning.

Keywords: strategic control; strategy; innovation; negotiation; planning.

Resumo: Para o planejamento de uma estratégia eficaz e eficiente, capaz de produzir os resultados desejados, é necessário que todas as partes que interagem com o processo estratégico militar estejam cientes da importância da negociação e de como ela serve como ferramenta para se chegar ao planejamento estratégico desejado. Com esta pesquisa não experimental, descritiva, com abordagem qualitativa e desenho transversal, busca responder ao questionamento: Como a negociação pode contribuir para o planejamento da estratégia militar? Para isso, primeiro são analisados os conceitos básicos de estratégia, inovação e negociação. Como um segundo ponto, o foco principal ou método direto em uma negociação bem-sucedida é identificado e, em terceiro lugar, é exposto como as negociações contribuem para o desenvolvimento estratégico.

Palavras-chave: controle estratégico; estratégia; inovação; negociação; planejamento. 
En la actualidad, la globalización y los desarrollos tecnológicos facilitan los procesos de interacción con diversas culturas. De igual forma, existe extensa información a la mano, con la que cada persona puede formularse diferentes perspectivas acerca de una misma situación o problemática. Al existir tan variados puntos de vista, se incrementan las diferencias o desacuerdos y esto hace que muchas veces los procesos de planeación estratégica sean ineficientes e impidan alcanzar los resultados deseados.

Como si lo anterior fuera poco, se suman, al proceso de planeación estratégica, la mala interpretación de conceptos clave en el proceso como lo son los términos innovación y estrategia, mismos que son el pilar para poder desarrollar estrategias nuevas y coherentes con los resultados deseados.

En los últimos años, se ha observado la forma en que muchos profesionales, con y sin experiencia en la planeación estratégica, interpretan estos conceptos, demostrando que no tienen una idea clara y concisa al respecto (Cardillo \& Szafranski, 2015). Esta situación, aunada a la poca capacidad de los profesionales responsables para llegar a acuerdos de forma efectiva, es precisamente la que interfiere en el éxito o fracaso de una innovación estratégica.

En este punto se debe aclarar que no se habla únicamente de innovaciones estratégicas en las compañías civiles, sino también en las unidades militares. Es importante preocuparse por entender que el presente artículo envuelve los términos correspondientes a innovación, estrategia y negociación, ya que a partir de allí será posible entender la forma en la que el poder innovar el método de negociación trae resultados positivos relacionados a la planeación estratégica.

Desde cualquier punto de vista, en todo lo relacionado con el proceso de negociación para la planificación de la estrategia, es importante tener una idea que busque el ganar-ganar de todas las partes involucradas. En relación al aspecto ganar-ganar, Covey (2003) menciona que:

Ganar-ganar significa que los acuerdos o soluciones son mutuamente benéficos, mutuamente satisfactorios.
Ganar/ganar ve la vida como un escenario cooperativo, no competitivo. La mayoría de las personas tiende a pensar en términos de dicotomías: fuerte o débil, rudo o suave, ganar o perder. Pero este tipo de pensamiento es fundamentalmente defectuoso. Se basa en el poder y la posición y no en principios (p. 129).

Se debe entender que, al desarrollar el proceso de planeación estratégica, interactúan muchas personas y hay que ser capaz de planear de manera efectiva, ya que como bien lo comentan Jones y George (2009): "La planeación es el proceso que usan los gerentes para identificar y seleccionar las metas y actividades apropiadas para una organización" (p. 263). Si no se planea de forma efectiva, será complicado alcanzar los objetivos propuestos.

Los directores de estrategia también se apoyan en la planeación efectiva para visualizar el entorno de una manera más fácil, ya que, cuando se habla de planeación estratégica, siempre es necesario ir un paso adelante de los competidores o enemigos. Con relación a esto último, es claro que quienes tienen la habilidad de observar ampliamente su entorno son mejores para obtener ventaja de las situaciones que acontecen (Mintzberg, 1989).

Este artículo consta de tres etapas: (1) se analizan los conceptos básicos de estrategia, innovación y negociación con la intención de introducir en contexto al lector, facilitándole el entendimiento del contenido de las siguientes etapas; (2) se identifica el enfoque primordial o método directo en una negociación exitosa, para ampliar el entendimiento del lector en el ámbito efectivo de la negociación y (3) se expone la forma en que contribuyen las negociaciones en el desarrollo estratégico.

Al terminar de leer este artículo, el lector podrá comprender la manera en que la negociación contribuye a la planeación estratégica militar; así como la importancia que conlleva el desarrollar estrategias militares innovadoras y el liderazgo estratégico, ya que este último contribuye a una mejor comunicación que, a su vez, permite alcanzar una planeación más efectiva (Jones \& George, 2009). 


\section{Antecedentes}

En la actualidad, el entorno social y profesional es cada vez más exigente y menos tolerante. En el día a día es difícil prestar atención a detalles operativos y ponerse de acuerdo con respecto a diversas situaciones; "muchos profesionales civiles y militares se quejan de las distracciones que sufren mientras ejecutan sus tareas, se sienten agobiados y tratan de ejecutar sus acciones de forma tal que les sea posible cumplir con lo encomendado y lo que es verdaderamente importante" (Y. Castillo \& F. Franco, comunicación personal, 9 de marzo del 2021).

Este correr diario distorsiona los conceptos clave y las acciones concretas que se podrían ejecutar para innovar procesos, estableciendo estrategias claras, bien fundamentadas y aceptadas de forma voluntaria por todos los involucrados.

Los oficiales militares deben preocuparse por tener claro qué envuelve a cada concepto y qué herramientas pueden ser utilizadas para la puesta en práctica de estrategias innovadoras que repercutan en una ventaja competitiva en el campo que desarrollen sus actividades.

Hasta el momento, quizás algunos no han visualizado el proceso de innovación en áreas fuera de la tecnología y se han mantenido al margen de esta, viéndola como algo ajeno a su entorno.

Al hablar con los responsables de los procesos estratégicos, varios carecen de la capacidad para visualizar, de forma concreta, los campos de aplicación en los que se pueden ejecutar procesos de negociación e innovación estratégica dentro de sus unidades militares. Muchos de estos oficiales también faltan de la habilidad para canalizar información que les permita dar solución a una problemática y generar propuestas para estrategias más efectivas a partir metodologías como la Mejor Alternativa a un Acuerdo Negociado (MAAN) o la Best Alternative to a Negotiated Agreement (BATNA), en inglés.

Por esta razón, es necesario contribuir a la ciencia con artículos que permitan aclarar de mejor manera la importancia de la negociación y el papel que esta juega en el proceso de planeación estratégica, aportando información valiosa que contribuya a una estructuración estratégica más amigable con los procesos de mejora continua.

\section{Metodología}

Para el caso específico de esta investigación no experimental de diseño transversal, Hernández et al. (2014), mencionan que los "diseños de investigación transeccional o transversal recolectan datos en un solo momento, en un tiempo único" (p. 154), en este caso, hace referencia al de esta investigación.

De forma concreta, para este estudio se aplica el enfoque cualitativo y de tipo descriptivo, por lo que se pretende describir, en todos sus componentes principales, la manera en la que la negociación contribuye en la planeación de la estrategia militar.

El proceso comienza con la revisión y el respectivo análisis de un máximo de 30 fuentes bibliográficas seleccionadas al azar y que están relacionadas con la estrategia, la innovación, la planeación estratégica y la negociación. El total de las fuentes consultadas no están estrictamente relacionadas al ámbito militar, dada la naturaleza de este artículo y la idea que el autor desea transmitir al lector.

Para el caso específico del tema negociación, se presta una mayor atención y valor a la literatura desarrollada por Fisher y Ury (1985), dada la importancia, el impacto, el valor y el prestigio que estos autores ostentan, así como por la naturaleza de la materia que, a pesar de los años, no se altera como ocurre con la información referente a tecnología u otras de evolución científica constante.

Para el caso de las entrevistas, se procedió con un muestreo no probabilístico al azar. Se realizaron cuatro a miembros del ejército de los Estados Unidos de América, ubicados en las bases de Okinawa, Japón. Para la elección de la muestra, los factores tomados en consideración fueron que, por un lado, los oficiales militares contaran con conocimiento y experiencia en la temática de este artículo y, por el otro, que fueran cercanos y de fácil acceso para las entrevistas. 
Es a partir del análisis de esta literatura y de las entrevistas que es posible desarrollar las herramientas que se exponen en el presente trabajo, así como dar respuesta a la pregunta de investigación: ¿cómo la negociación puede contribuir a la planeación de la estrategia militar?

\section{El contexto de la estrategia y la innovación}

\section{La estrategia}

Schmitt et al. (2016) mencionan que, sin lugar a dudas, el tema referente a estrategia ha experimentado un creciente volumen de investigaciones, lo que ha contribuido, paradójicamente, a una mayor divergencia entre las perspectivas conceptuales y los ámbitos empíricos, así como a los consensos de lo que es la estrategia como tal. Se debe tener claro que muchas veces "hablar de estrategia se puede convertir en una torre de babel en la que muchos expresan ideas y quieren hacerlas valer, pero que nadie entiende a nadie" (Contreras, 2013, p. 155). Por lo anterior, es importante hacerse una idea clara de lo que es en sí la estrategia.

Para empezar, Mintzberg (1989) menciona que las estrategias son "planes para el futuro y patrones del pasado" (p. 30). En otras palabras, aprender de las experiencias pasadas para planear el nuevo accionar en el futuro. Lo que se puede rescatar de esto es que aprender de las experiencias ayuda positivamente en el desarrollo del entorno, incluyendo, por supuesto, las acciones militares. Relacionado con esto último, Chandler (1962) comenta que "el crecimiento estratégico resultó de una conciencia de las oportunidades y necesidades, creadas por el cambio de población, ingresos y tecnología, para emplear los recursos existentes o en expansión de manera más rentable" (p. 15).

Por otro lado, Kenichi (1982) explica que la estrategia "es lograr las condiciones más favorables para uno mismo, juzgando precisamente el momento adecuado para atacar o retirarse y siempre evaluando los límites del compromiso correctamente" (p. 13). Lo anterior se puede interpretar como la capacidad o habilidad de analizar muy bien los factores internos y externos de la unidad militar para, mediante el elemento sorpresa, atacar a los enemigos, logrando así una ventaja competitiva en la zona de guerra.

Analizadas estas definiciones y, después de realizar las entrevistas a oficiales militares, podemos decir que estrategia es la habilidad para analizar los factores internos y externos de la fuerza militar con la intención de dirigir acciones concretas que busquen alcanzar una meta claramente establecida. Lo anterior, a través del elemento sorpresa en contra de la competencia, logrando así, una ventaja competitiva en la zona de guerra en que se desempeña.

En resumen, "estrategia es la acción de sorprender, confundir o engañar a nuestros competidores con ayuda de la información de la cual disponemos, esto, con la intención de alcanzar una meta” (Segura, 2020, p. 24).

\section{La innovación}

En la última década, algunos investigadores se han interesado enormemente en las innovaciones militares que algunos de los principales ejércitos han desarrollado para optimizar sus operaciones y habilidades. Al respecto, Fernández-Osorio et al. (2019) creen que:

Este interés ha generado un gran volumen de estudios publicados que tienden a comprender las transformaciones militares como un proceso de innovación, que busca preparar una fuerza militar adecuada y apta para enfrentar las amenazas y desafíos de un mundo globalizado (p. 75).

Cuando se habla de innovación, generalmente se asocia a la del producto tecnológico, olvidando que no es solo esto. Según la Organización para la Cooperación y el Desarrollo Económico (OCDE) y la Oficina Europea de Estadística (EUROSTAT), los tipos de innovaciones que se pueden identificar son: "Innovaciones de producto, innovaciones de proceso, innovaciones organizativas e innovaciones de mercadotecnia" (OCDE \& EUROSTAT, 2005, p. 23). 
La innovación no puede ser únicamente enfocada en términos de tecnología del producto o como un tema aislado de las compañías civiles. Por el contrario, también debe tenerse presente que las operaciones militares pueden y deben estar sujetas a esta.

Es indispensable el destinar recursos para las innovaciones en términos de operaciones militares (proceso, organización y mercadotecnia), ya que por sí solas "no constituyen solamente un factor de apoyo para la innovación de producto y proceso; ellas mismas pueden influir considerablemente en los resultados" (OCDE \& EUROSTAT, 2005, p. 18).

Por otra parte, en la actualidad se observa que la globalización, impulsada por los desarrollos tecnológicos, ha generado un mayor y fácil acceso a la información, lo que fomenta una competitividad internacional y nuevas formas de administración. De esto se puede visualizar que el conocimiento y el acceso a la información se consideran cada vez más un impulsor del crecimiento económico y de la innovación (OCDE \& EUROSTAT, 2005).

La globalización ha provocado que los actores del entorno económico tengan mayores facilidades para apreciar las diferencias en las cualidades de productos y servicios, no solo en el ámbito local, sino también en el internacional, lo que ha obligado a las compañías a innovar cada día, de una manera más eficiente.

Dicho lo anterior, se tiene un panorama más claro de la situación en muchas unidades militares. La competitividad que a diario se incrementa entre estas, impulsa la necesidad de innovar en diferentes vertientes, las cuales, directa o indirectamente, también vienen a repercutir en los procesos o métodos militares utilizados en la actualidad. Al existir avance tecnológico, los procesos o métodos de trabajo y de estrategia se ven en la necesidad de innovar también.

La acción de innovar no es solo para hacer cosas diferentes y mejores. También es necesario saber visualizar que "la expansión de la actividad innovadora en las economías incide cada vez más en la distribución regional del ingreso" (Organización Mundial de la Propiedad Intelectual [OMPI], 2019, p. 5). La actividad militar debe ser vista como un instrumento que tiene la capacidad de alterar el orden económico de una región.
Al igual que las compañías civiles, las fuerzas militares de hoy deben poder innovar para lograr diferenciarse, recordando que "diferenciación significa recorrer el camino hacia la exclusividad absoluta construyendo dimensiones únicas en el sector industrial" (Pulgarín \& Rivera, 2012, p. 97).

El cambio debe ser ágil y rápido, ya que "solo para mantener su posición relativa, una compañía debe avanzar a través de un crecimiento y cambio continuos. Para mejorar su posición, debe crecer y cambiar al menos dos veces más rápido que eso" (Ansoff, 1957, p. 113), lo que impulsará la búsqueda de la excelencia en las actividades que se ejecutan y, directa o indirectamente, guiará hacia la innovación continua.

En concordancia con lo que menciona Serna (2008), en todo este proceso se necesitan líderes militares que no tengan temor al cambio y que sepan motivar a los miembros su equipo para alcanzar los objetivos estratégicos establecidos, siempre manteniendo una dirección clara.

Bien sean colaboradores militares o la fuerza militar como tal, se debe estar en la búsqueda constante de la ventaja competitiva por medio de la innovación, ya que como bien lo menciona Segura (2020), la "ventaja competitiva es aquella que, por una u otra razón, nos hace únicos en el mercado, con características que no son tan fáciles de igualar por nuestros competidores" (p. 50). Entendiendo al "mercado" como al espacio militar donde se desarrollan las actividades y a los "competidores" como a los adversarios o enemigos. Vale la pena resaltar que "la renovación estratégica es igualmente importante para permitir la innovación y la evolución" (Schmitt et al., 2016, p. 14).

Así las cosas: ¿en qué aspectos podrían innovar las Fuerzas Militares? En la Biblioteca Virtual Universal (2003), es posible encontrarse con Sun Tzu y su libro El arte de la guerra, en el cual se menciona que existen cinco factores fundamentales para valorar en la guerra: la doctrina, el tiempo, el terreno, el mando y la disciplina. Segura (2021) comenta que "cada uno de estos cinco elementos está sujeto a la innovación estratégica por la simple y llana razón de que existen procesos, herramientas o equipos tecnológicos que podrían ser mejorados" (p. 18). 
Así, se podría innovar en los incentivos motivacionales para que permitan adoctrinar (la doctrina) de mejor manera a las tropas; innovar en los equipos, maquinaría u otras herramientas utilizadas en terrenos (el terreno) complejos, facilitando las estrategias de ataque; innovar en las estrategias que ayuden a ejecutar operaciones militares en condiciones climáticas (el tiempo) adversas para los enemigos pero beneficiosas para su propia unidad, dada la innovación realizada en el proceso, la herramienta, el equipo, entre otros casos.

A través de los conceptos de estrategia e innovación se puede visualizar que en el desarrollo de estrategias o procesos de innovación es necesario para interactuar con otros individuos, ya sean parte del equipo de trabajo, o no, lo que da una idea de cómo la negociación contribuye en el proceso de la planificación de la innovación estratégica.

\section{¿Qué son las negociaciones?}

No está de más el tener presente que varios líderes militares han sido capaces de entender y alcanzar con éxito el ámbito de las negociaciones mientras que a otros no les ha sido posible (Cronin, 2015), por lo que es necesario exponer, en este apartado, la teoría referente al tema de las negociaciones. Así el lector podrá asociar o vincular más fácilmente la negociación con la planeación estratégica, dando respuesta a la pregunta de investigación.

Para Fernández-Osorio et al. (2019), "existe una percepción común en la literatura de que una transformación militar puede ser desfavorable si los líderes carecen de las habilidades necesarias para asegurar su cumplimiento y continuidad" (p. 76). Como podrá verse a continuación, para poder asegurar ese cumplimiento y su respectiva continuidad, es necesario negociar.

Un tema que se encuentra muy relacionado con las negociaciones es la inteligencia emocional, la cual "es la capacidad de entender y manejar las emociones y los estados de ánimo propios y de los demás" (Jones \& George, 2009, p. 90); de lo que se infiere que, si no se saben controlar de forma correcta emociones o sentimientos como la furia, la tristeza, la duda, entre otros, se puede dar lugar a la falta de capacidad para dirigir la negociación en la cual se esté involucrado, perdiendo así, muchas oportunidades para llegar a un acuerdo satisfactorio.

Es fundamental entender y recordar que cada persona, a partir de sus creencias o experiencias, interpreta una situación de diversas maneras y que, también, cualquier individuo, al igual que su contraparte, tiene muchos problemas girándole en la cabeza. Estas situaciones podrían desembocar en la incapacidad de llegar a desarrollar acuerdos beneficiosos para ambos. En todo esto, es indispensable tener la capacidad de comunicarse de manera efectiva (Fisher \& Ury, 1985).

Hay que ser precavido para no encerrarse en percepciones, entendiendo, también, a la contraparte. Los sentimientos se generan porque alguna situación especial los ocasiona (son el efecto de una causa), por lo que Covey (2003) indica que la "gente se siente cada vez más impotente y privada de su autocontrol, alejada de su vida y de su destino. Culpa a fuerzas externas - a otras personas, a las circunstancias, incluso a los astros- de su propia situación" (p. 48). Por esta razón es importante saber identificar las causas que provocan los sentimientos, para, asimismo, manejarlos de forma efectiva para el beneficio de todos.

En línea con la idea anterior, Harvard University (2020) indica además que "como negociador experimentado, sabe que debe prepararse para las conversaciones, evaluando los intereses de cada parte y las opciones de no negociar, imaginando posibles acuerdos, teniendo en cuenta la personalidad y la cultura" (p. 8).

Es indispensable estudiar a la persona con quien se negociará, ya que, dependiendo del perfil del negociador que se confeccione, se podrá tener un panorama más claro de muchos sentimientos o situaciones que podrían salir a flote mientras se lleva a cabo el proceso.

El poder entender los sentimientos de otras personas, sus culturas y sus personalidades amplifica la creatividad y ayuda a crear propuestas que vayan más acorde con los ideales de su contexto, incrementando las posibilidades de acuerdos efectivos que permitan alcanzar los objetivos propuestos en el plan estratégico. 
El manejo de las emociones es fundamental para garantizar el control de una situación, es decir, que si se pueden controlar, entendiendo a las de la contraparte, será más fácil elaborar propuestas efectivas $y$, por supuesto, se establecerán procesos estratégicos aceptados de forma voluntaria y colectiva.

Con la intención de explicar mejor la manera en la que las negociaciones desempeñan un papel importante en la estrategia, se presenta la siguiente información:

Se puede mencionar que el análisis de una situación, el juicio y la negociación son aspectos fundamentales que los responsables estratégicos pueden utilizar para la efectiva toma de decisiones (Mintzberg, 1989).

Para realizar el proceso de planeación estratégica, el cual está estrictamente relacionado con la capacidad de planear, de forma tal que sea posible llegar a los resultados de forma efectiva, previendo imprevistos que puedan alterar o neutralizar la estrategia (Villacis, 2014), se requiere del análisis de la información interna y externa de la unidad militar, la cual, una vez formulada hará uso de procesos de negociación, esto con la intención de acordar cuál o cuáles estrategias serán lideradas por la gerencia estratégica (líder militar a cargo de la ejecución estratégica). De la misma forma, la gerencia estratégica necesitará, con la intención de desempeñar un liderazgo eficiente, negociar con varios actores involucrados durante el proceso de asignación de recursos en su camino por alcanzar los resultados deseados.

Para continuar, es fundamental que se entienda qué es negociación, así como los principales elementos que envuelven su definición.

Cambridge University (s. f.) la define como "el proceso de discutir algo con alguien para llegar a un acuerdo"; mientras que la Real Academia Española (2019) se refiere a las acciones que se ejecutan para dar por concluido un acuerdo entre las partes.

Al analizar más definiciones de negociación, Shonk (2019) menciona que es la acción necesaria para ponerse de acuerdo con la contraparte acerca de una situación específica que no puede ser resuelta de forma independiente, y que, de igual manera, en caso de disponer de intereses o posiciones opuestas respecto de una misma situación, también es necesario negociar para poder llegar a un acuerdo.
Se concluye entonces que la negociación es el proceso mediante el cual dos o más personas, a través de ideas bien fundamentadas o sin fundamentar, interactúan entre sí con la intención de llegar a un acuerdo que sea aceptable para las partes que desean alcanzar un resultado dentro de un contexto específico.

En muchísimas ocasiones es necesario negociar. En la actualidad, las interrelaciones culturales que se experimentan traen consigo conflictos que es necesario solucionar a través de negociaciones. Por lo general, las personas quieren que se ejecuten las propuestas que ellos exponen y para poder persuadir, no queda más que desarrollar negociaciones efectivas (Fisher \& Ury, 1985). Prácticamente, todo lo referente a los conflictos o diferencias que existan en un entorno, al igual que la toma de decisiones, entre más de dos personas, necesitan hacer uso de la negociación.

El proceso de negociación es indispensable en la vida diaria, pues ayuda a dirimir, desde negociaciones personales simples, hasta decisiones de alto impacto para organizaciones o estados. Como bien lo comentan Fernández-Osorio et al. (2019), unos de los factores que contribuyen a potenciar el desarrollo de la unidad militar son las alianzas estratégicas, locales o internacionales, y su efectiva vinculación con otras organizaciones, además de las correctas políticas de operación. Para ser capaces de lograr lo anterior, es indispensable una negociación efectiva que contribuya al desarrollo de las actividades necesarias para alcanzar un plan estratégico funcional.

Una negociación exitosa puede significar un gran beneficio para diferentes participantes; prueba de ello, Abello (2006) nos menciona que:

Las negociaciones futuras van a girar en torno a las inversiones, pero, fundamentalmente, el tratamiento a la inversión extranjera directa (IED) en los países en desarrollo es también objeto de preocupación en la OMC, en el entendido de que esta IED es concebida como un medio para el progreso tecnológico, el acceso a los mercados y el desarrollo (p. 450).

Como se puede apreciar en este caso, las negociaciones relacionadas con la atracción de Inversión 
Extranjera Directa (IED), que pueden desarrollar diferentes países, repercutirá de manera directa en su población. Si no se ejecutan negociaciones coherentes y alineadas con la estrategia que se haya formulado, los resultados pueden ser negativos para sus habitantes debido a que estarán saliéndose de lo que, en teoría, fue planificado, generando descontrol e imprevistos en las operaciones.

Por otra parte, no se puede pasar por alto que cada persona perfila criterios bajo sus propias creencias, lo que hace que una misma situación sea vista o interpretada de muchas maneras, y a su vez, provoca diversos tipos de conflictos. Con respecto a este último punto, Jones y George (2009) comentan que un conflicto es una discrepancia o disputa que sale a flote cuando los objetivos, valores o principios de los grupos o equipos no están alineados.

Lo anterior no es una situación ajena al proceso estratégico, ya que al igual que en cualquier otro tipo de operación, está expuesto a situaciones de conflicto entre sus participantes. Estas diferencias pueden convertirse en posiciones, es decir, que cada uno de los participantes tome una idea o percepción y la considere como la única opción viable para un posible acuerdo, haciendo todo más difícil y, por ende, dificultando el proceso para la planeación estratégica.

Es importante enfocarse en cuáles son los verdaderos intereses de las partes involucradas, para asíllegar a una solución o acuerdo eficiente.

Todo lo referente a la industria de posiciones es un tema complejo, pues "cuanta mayor atención se presta a las posiciones, menor atención se dedica a satisfacer los intereses y preocupaciones subyacentes de las partes" (Fisher \& Ury, 1985, p. 13).

En la actualidad se dispone de dos tipos de negociaciones que, según Jones y George (2009), son:

Distributiva e integradora. [...] En la negociación distributiva las dos partes piensan que existe un "pastel" de recursos que tienen que dividirse. [...] En la negociación integradora, las partes piensan que pueden agrandar el pastel si conciben una solución creativa para el conflicto (p. 613).
Como se aprecia, hay dos tipos de negociación, la negociación distributiva o basada en posiciones dispone de dos enfoques que el negociador puede elegir, como lo son las "versiones dura y suave de la negociación basada en posiciones” (Fisher \& Ury, 1985, p. 16). Enfoques que según los mismos Fisher y Ury (1985) no ofrecen los resultados eficaces que la negociación integradora, también denominada negociación según principios o negociación con base en los méritos. Hay que tener en cuenta que, en algunos artículos o literatura, se conoce también como método de negociación de Harvard.

Dado lo anterior, se quiso plasmar por medio de las figuras 1 y 2 una idea gráfica de lo correspondiente a cada tipo de negociación.

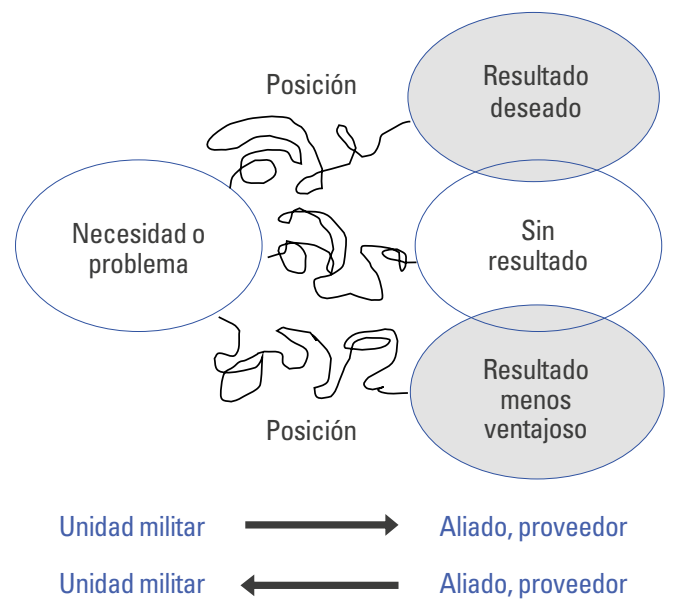

Figura 1. Negociación con beneficio unilateral Fuente: elaboración propia.

En la imagen anterior se aprecia el proceso de negociar con base en posiciones, de manera distributiva o bien, con beneficio unilateral. Las líneas enredadas representan que, cuando se negocia con base en este tipo de negociación, es muy difícil llegar a un resultado satisfactorio de manera rápida. Por lo general, el desgaste de los negociadores o partes involucradas es mayor que en el otro tipo de negociación.

Si se enfoca en posiciones, se caerá en la redundancia, desgastándose más de lo necesario, lo cual, según la imagen anterior, puede conducir la negociación por tres caminos: 
- Podría llegarse al resultado deseado, pero de una manera muy desgastante y sin la garantía de una buena relación con la contraparte a futuro.

- Después de invertir mucho esfuerzo, es posible no lograr ningún resultado o acuerdo.

- Se podría llegar a un resultado o acuerdo menos ventajoso del que se pudiera haber llegado por medio del otro tipo de negociación.

En la negociación de beneficio unilateral, o como realmente se conoce, negociación distributiva o con base en posiciones, generalmente, las partes involucradas buscan el juego de suma cero: yo gano y usted pierde. Eso explica las flechas de un solo sentido en la parte de abajo de la imagen.

Con relación a este tipo de negociación, Covey (2003) indica que "las personas del tipo gano-pierdes son proclives a utilizar la posición, el poder, los títulos, las posesiones o la personalidad para lograr lo que persiguen" (p. 129). En otras palabras, es la que más se aprecia en la gente ubicada en cargos superiores para con sus subordinados o en las personas con personalidad de superioridad.

Este tipo de negociación también se conoce algunas veces como regateo, lo cual hace que los miembros involucrados se comprometan más con sus posiciones o puntos de vista, generando el sentimiento de estar a la defensiva y de querer contraatacar al más mínimo gesto o acción que le haga pensar que su contraparte quiera tirarle abajo su posición. Reafirmando lo anterior, Fisher y Ury (1985) nos comentan que "cuando se regatea con base en las posiciones, los negociadores tienden a encerrarse dentro de ellas. El negociador, mientras más aclara su posición y más la defiende contra los ataques, más se compromete con ella" (p. 13), lo que desencadena en que las relaciones de los negociadores se deterioren o que hasta se rompan. Con relación a esto último, Harvard University (2020) informa que:

En las negociaciones puntuales en las que no espera volver a cruzarse con su contraparte negociadora, puede importar poco si no le gustas. Pero la mayoría de las negociaciones, por supuesto, conllevan al menos la posibilidad de futuras interacciones. Si está satisfecho con su experiencia en la negociación de un auto nuevo, por ejemplo, puede recomendar el concesionario a sus amigos o elegir regresar cuando llegue el momento de comprar su próximo auto (p. 6).

Si en las organizaciones militares se desea formular estrategias que tengan un impacto a futuro, es necesario desarrollar negocios que generen buenas relaciones entre todas las partes, ya que no se gana nada con usar la negociación distributiva con un proveedor el día de hoy para obtener un precio más bajo en comparación a otros proveedores si, dentro de dos años, este, por ceder a la primera, sacrificó calidad o desapareció por la poca utilidad que generaba dicha relación comercial.

Sin generar buenas relaciones en una negociación, no se podrá pretender tener la seguridad de continuar bajo la misma línea en un futuro. Sin buenas relaciones no hay futuras negociaciones $y$, evidentemente, esto afectará la planeación estratégica.

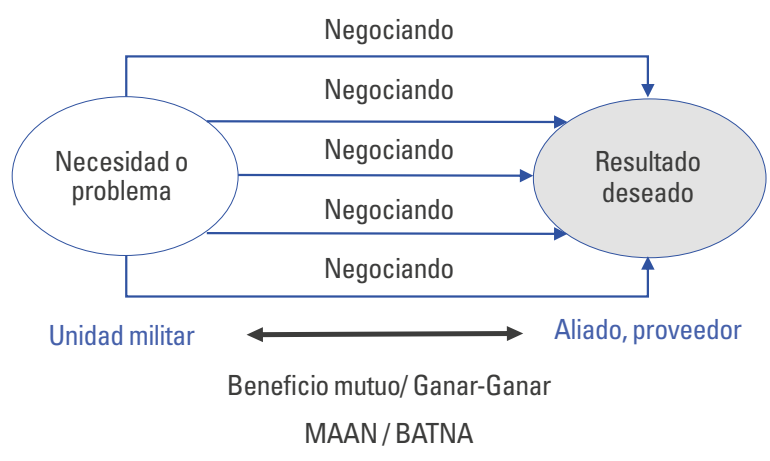

Figura 2. Negociación con beneficio multilateral Fuente: elaboración propia.

En esta imagen se pueden apreciar grandes diferencias con respecto a la figura 1. Hay que notar, primero, las líneas rectas que conectan directamente la situación (necesidad o problema) con el resultado deseado. Esto indica que, si se enfoca en negociar haciendo uso del método de negociación de Harvard, el proceso será más directo, rápido y menos agotador que con el método de negociación distributiva. De igual manera, al hacer uso del segundo método, se 
tendrán muchas más posibilidades de estar en una negociación ganar-ganar, en la cual, todas las partes involucradas obtienen más de lo que hubieran podido haber imaginado. De ahí la presencia de esa flecha en ambos sentidos, ya que la unidad militar puede ganar igual que el aliado o el proveedor. Al respecto del punto de vista ganar-ganar, Covey (2003) menciona que:

Ganar/ganar significa que los acuerdos o soluciones son mutuamente benéficos, mutuamente satisfactorios. Ganar/ganar ve la vida como un escenario cooperativo, no competitivo (p. 129).

Aportando más sobre este aspecto, Fisher y Ury (1985) mencionan que se negocia:

Con la intención de conseguir algo que no se podría conseguir de otra manera. Y por supuesto, para poder ser conscientes de que se puede aceptar y que no aceptar en una propuesta. El conocer objetivamente hasta donde se puede ceder y que se puede solicitar que ceda la contraparte, ayudara a obtener un mayor beneficio para las partes (p. 85) .

En ese sentido, el MAAN o BATNA podría interpretarse como la herramienta que permite, de manera planificada y objetiva, determinar si una propuesta puede ser aceptada o rechazada; además de que prevé lo que podría hacerse de no llegar a un acuerdo.

Luego del análisis de la literatura referente a negociación, en la figura 3 , que se muestra a continuación, se presenta el proceso que se debería seguir para lograr elaborar el MAAN o BATNA deseado, en una negociación.

Dicho diagrama también puede considerarse como un proceso de planeación, asociándose al sentido que viene a tener el plan estratégico. En otras palabras, esta metodología sirve de soporte para varias actividades vinculadas al plan estratégico, ya que como se pudo ver, para el desarrollo de la planeación estratégica se necesita negociar, llegando a acuerdos entre las partes responsables de ejecutar las actividades vinculadas a la estrategia.

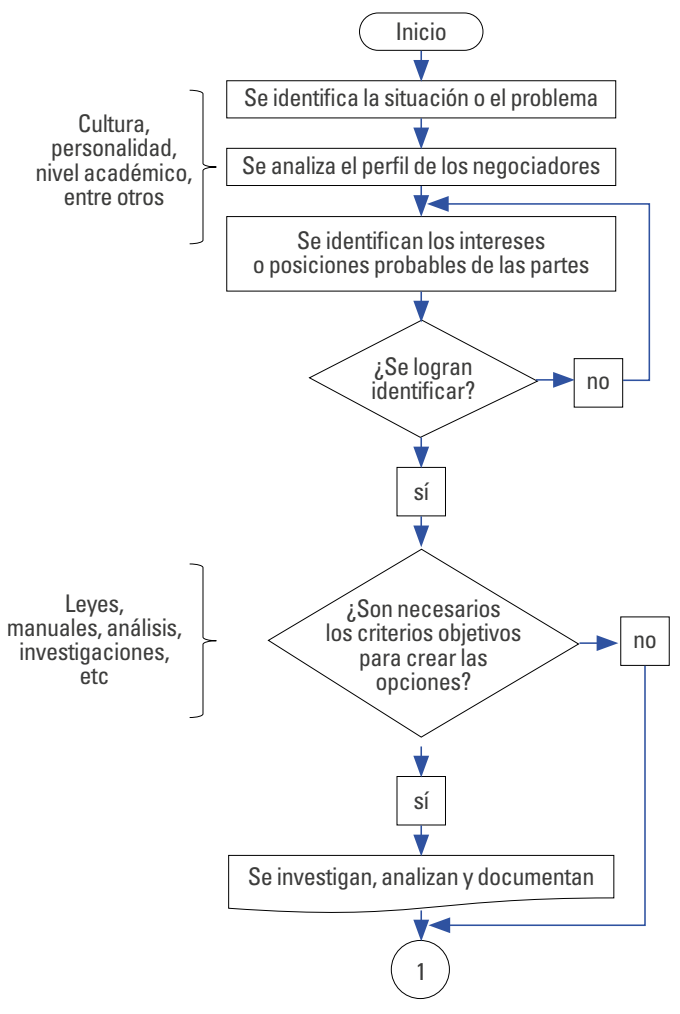

Figura 3. Preparación del MAAN o BATNA

Fuente: elaboración propia.

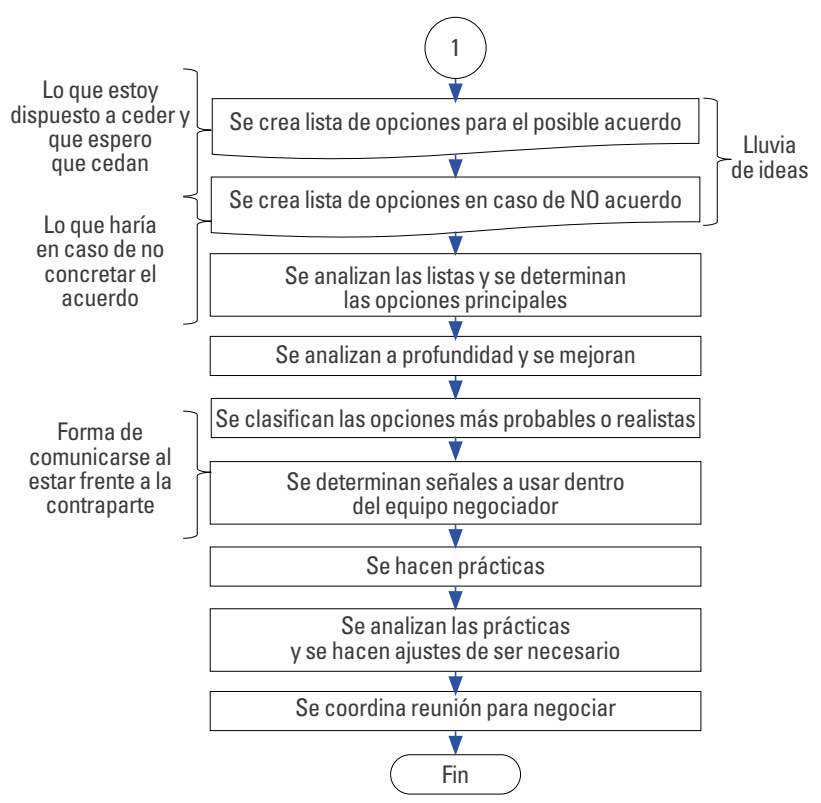

Figura 4. Preparación del MAAN o BATNA (Continuación)

Fuente: elaboración propia. 
Como se mencionó, el diagrama anterior es una forma de planeación estratégica y se usa para formular el MAAN o BATNA para ser puesto en ejecución cuando se esté frente a la contraparte, buscando los resultados estratégicos deseados o la Mejor Alternativa al Acuerdo Negociado (MAAN), en ese momento.

Por otra parte, es necesario tener presente que "es importante buscar formas de equilibrar su deseo de alcanzar un objetivo elevado con la necesidad de construir una buena relación" (Harvard University, 2020, p. 6), pues las buenas relaciones podrán permitir un mejor rendimiento en el desarrollo del proceso estratégico.

\section{Enfoque primordial o método directo en una negociación exitosa}

En muchos aspectos o procesos de la ciencia se debe tener una metodología clara y concisa que garantice el éxito de una acción, actividad, proceso o procedimiento: lo mismo sucede al momento de negociar.

Solo para darse una idea, con el diseño de un servicio o producto, se hace posible la interacción de diversos profesionales, trayendo consigo un rico intercambio de ideas y conocimientos, así como de conflictos. Al respecto Chase et al. (2009) indican que:

Diseñar un producto que complazca al cliente es todo un arte. Fabricar el producto es una ciencia. Llevar el producto desde el diseño hasta el cliente es administración. Los fabricantes de clase mundial son excelentes para lograr una integración expedita y flexible de estos procesos. Una llave para hacerlo es el trabajo en equipo, no solo de parte de marketing, desarrollo de productos, producción y distribución, sino también de parte del proveedor y el cliente (p. 213).

Todo lo que involucra el proceso de desarrollo y entrega de un producto o servicio conlleva negociación, lo que obliga a conocer más sobre el enfoque principal o método directo que se debe tener o aplicar a la hora de negociar un acuerdo con los proveedores, colaboradores, aliados u otros.
Dicho lo anterior, existen varios métodos de negociación; sin embargo, es necesario que como estratega se reconozca aquel que busque el beneficio real y eficiente de las partes involucradas. Al respecto, el método de negociación integrativo o de negociación de Harvard puede ser la mejor alternativa. Este es muy eficiente debido a que sus pilares permiten alinearse fielmente a principios que guían durante una negociación, haciendo más fácil y eficiente el proceso.

Algunos autores, como Paz (2004); Sarkis y Ocampo (2015); Ogliastri (2001) o Shonk (2019), mencionan elementos que sirven como herramientas para mejorar el proceso de negociación. Estos se resumen en los cuatro puntos de la siguiente figura:

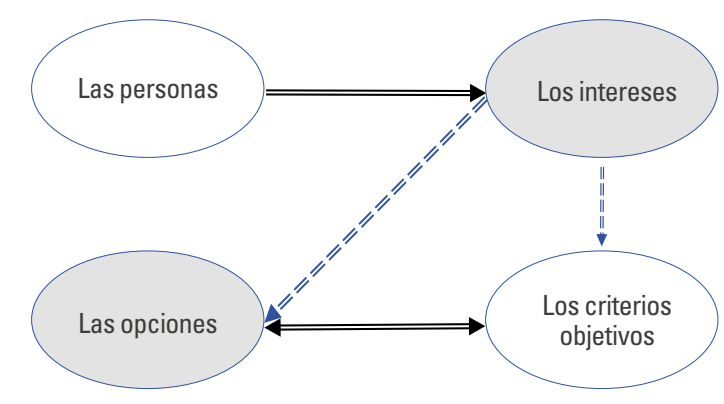

Figura 5. Elementos que definen un método directo de negociación Fuente: elaboración propia a partir de datos de Paz (2004, p. 91) y Fisher y Ury (1985, p. 17).

Antes de describir cada uno de los elementos de esta imagen se puede decir que, para iniciar el proceso de la negociación se debe analizar muy bien a las personas que estarán involucradas e identificar y confirmar los intereses, lo que permitirá desarrollar ideas de propuestas (opciones). De las ideas que se generen, se debe verificar si existen criterios objetivos que las respalden, para poder fundamentarlas. También, de los intereses, se puede saltar al los criterios objetivos para analizar la información disponible y diseñar las propuestas (opciones). Por esta razón existen las flechas punteadas y la flecha de doble sentido entre estos elementos. En otras palabras, primero se crean las opciones de propuesta y se respaldan con los criterios objetivos. Primero se recopilan los criterios objetivos y, a partir de acá, se crean las propuestas. 
A continuación, se detallan cada uno de los elementos para el método directo de negociación, que permitirán tener una perspectiva más clara de los componentes críticos a considerar, buscando alcanzar los resultados deseados en el proceso de la planeación estratégica.

\section{Las personas}

En toda negociación se deben separar a las personas del tema que se está tratando: una cosa es el negociador y otra el problema que se tiene en frente. Es importante hacerle ver a la contraparte que se le respeta como ser humano. También, se debe tener en cuenta que "cuando la confianza es mutua, las prácticas tienden a ser transparentes y así se logra generar valor para beneficio de las partes" (Sarkis \& Ocampo, 2015, p. 145). De igual manera, las partes que interactúan en el proceso deben verse a sí mismos como equipo que ataca un problema y no como competidores que buscan un ganador independiente, debe existir tolerancia entre los participantes que desarrollan una negociación (Paz, 2004, p. 92).

\section{Los intereses}

Es necesario conocer a profundidad el interés de la contraparte, identificando lo que son las posiciones para buscar mejores alternativas para el cierre del acuerdo. Como comenta Bermúdez et al. (2019), de acuerdo con los interés del responsable de la toma de decisiones en el proceso de negociación, se podrían ver socavados los objetivos de las estrategias y políticas de Estado, provocando que los militares experimenten un ambiente indeciso y confuso frente a las acciones que deberían haber ejecutado en las negociaciones u otras relacionadas con la planeación estratégica. Si no existe claridad en los intereses de una negociación, será difícil llegar a desarrollar acuerdos eficientes, lo que podría afectar el proceso de planeación estratégica.

\section{Las opciones}

Es importante pensar en múltiples opciones para llegar al acuerdo, no importa que parezca una propuesta poco atractiva, si no afecta en ningún aspecto los intereses propios, se debería mencionar y así quizás se logre algo, o bien, surja una nueva idea.

Como bien lo mencionan Sarkis y Ocampo (2015), es posible llegar a generar opciones importantes cuando existe un amplio interés de las partes en el cierre del acuerdo y disponen de una mentalidad abierta, por lo que es indispensable preparar la negociación antes del encuentro. En otras palabras, preparar el MAAN o BATNA es una parte fundamental de la estrategia.

\section{Los criterios objetivos}

En cada palabra que se mencione o idea que se proponga se deben establecer bases objetivas. Todo debe estar bien respaldado para evitar salidas inesperadas y poco fundamentadas de la contraparte: no se debe hablar por hablar. En relación con esto, Paz (2004) comenta que "mediante los [criterios objetivos] se define si los intereses que proclaman ambas partes son justos, en comparación con un patrón de referencia externo e imparcial" (p. 93). Iniciar la búsqueda de criterios objetivos, en algunos casos, podría generar ansiedad por llegar a datos que sean relevantes en la negociación que se está desarrollando, lo que nublaría la mente y haría caer en error a quien esté negociando. Al respecto, Harvard University (2020) menciona que "la ansiedad es la emoción más comúnmente experimentada antes de una negociación, más que la emoción, la tristeza, la calma o la ira” (p. 15). Esta situación podría generar que los responsables tomen decisiones incorrectas que puedan afectar el desempeño de los resultados deseados. Una buena negociación incrementa la posibilidad de desarrollar estrategias efectivas y con gran proyección de éxito a largo plazo.

\section{¿De qué forma sirven las negociaciones en el desarrollo estratégico?}

Para experimentar un desarrollo efectivo en cualquier ámbito, las personas deben saber trabajar en equipo, tener una comunicación efectiva y ser capaces de llegar a acuerdos fundamentados en el ganar-ganar, ya que esta será la única forma en la que el equipo se 
mantendrá unido y luchará por alcanzar los resultados deseados en común.

Si los estrategas no son capaces de negociar acuerdos efectivos que sean para beneficio de todas las partes involucradas en el proceso estratégico, los actores de este podrían visualizar un resultado ganar-perder que los pondría a la defensiva y haría que el ambiente se torne tenso y que, por ende, se deterioren las relaciones del equipo de trabajo. Con relación a lo anterior, nuevamente Covey (2003) recalca que:

Ganar-ganar, en cambio, se basa en el paradigma de que hay mucho para todos, de que el éxito de una persona no se logra a expensas o excluyendo el éxito de los otros. Ganar-ganar se basa en la creencia de que existe una tercera alternativa. No se trata de tu éxito o el mío, sino de un éxito mejor de un camino superior (p. 129).

El estratega negociador debe tener la habilidad de poder persuadir a las partes involucradas en el proceso estratégico, como lo pueden ser aliados, proveedores, colaboradores u otros, logrando acuerdos que le permitan a la organización militar alcanzar sus metas y desarrollar de forma armoniosa su proceso de planeación estratégica.

De lo expuesto anteriormente por Covey (2003), también se puede inferir un tono motivacional, entendiendo así que la "motivación puede definirse como las fuerzas psicológicas que determinan la dirección del comportamiento de una persona en una organización, su nivel de esfuerzo y su nivel de persistencia ante los obstáculos" (Jones \& George, 2009, p. 463). Tener la habilidad de motivar efectivamente a su gente para alcanzar resultados estratégicos también es parte del negociador. Si no se cuenta con colaboradores motivados, posiblemente sus mentes estén más cerradas a posibles soluciones o a acuerdos que puedan traer beneficio para todos.

De lo discutido hasta el momento en este artículo, y con el objetivo de dar respuesta a la pregunta de investigación, se puede indicar que la negociación contribuye en la planeación estratégica mediante la búsqueda incansable de soluciones efectivas a los problemas que se presenten durante todo el proceso estratégico. Si a los conflictos o diferencias presentes en el proceso de planeación de la estrategia militar no se les busca una solución efectiva, es imposible pretender que se alcancen los resultados estratégicos deseados.

\section{Conclusión}

Con el estudio de este trabajo pretendemos disponer de una perspectiva diferente del papel que juega la negociación en la estrategia. Ha sido posible identificar los tipos de negociación que existen y entre estos, la mejor opción para aplicar.

En muchas situaciones, algunos profesionales consideran que son pésimos negociadores o que no tienen la habilidad necesaria para llegar a buenos acuerdos; sin embargo, para Shonk (2019) existen esperanzas para las personas que no son negociadores natos, ya que puede decirse que las investigaciones realizadas muestran consistentemente que la mayoría de las personas pueden mejorar significativamente sus capacidades o habilidades de negociación por medio de herramientas como la educación, la preparación (entendiéndose bajo un contexto de planeación) y la práctica.

Por otra parte, hay que tener en cuenta que la comunicación es uno de los principales componentes de la vida y, por supuesto, de la negociación. Nunca se podrá llegar a ningún lugar si no se es posible expresar o comunicar bien los intereses. La comunicación abre o cierra puertas, e igualmente, da acuerdos exitosos o fracasos. Siempre es importante escuchar a la contraparte para saber bien qué quiere dar a entender. Así mismo, es bueno repetir lo que dice para confirmar que lo que se entendió es lo que se quería transmitir.

Cuando se sabe escuchar bien a la contraparte, es esta la que da las respuestas que se querían encontrar para llegar a un acuerdo exitoso.

Por último, para dar respuesta a nuestra pregunta de investigación, se puede decir que esta lo hará dado que siempre existirá la necesidad de negociar 
con los participantes del proceso de planeación, por ejemplo colaboradores, proveedores, gobierno, aliados, entre otros.

Como bien lo menciona Bermúdez et al. (2019), "en las negociaciones, la participación de las Fuerzas Militares no solo fue determinante, sino garante del mismo proceso, lo cual se constituye en un modelo y quizá doctrina o experiencias novedosas para futuros procesos de negociación a nivel global” (p. 12).

Es a través de la negociación que será posible llegar a acuerdos entre las partes involucradas en el proceso del desarrollo de la estrategia militar, permitiendo ejecutar estrategias más innovadoras, conocidas y aceptadas por todos los actores involucrados en el proceso. Siempre se debe pensar en negociar bien; con buena comunicación; buen trato a las personas; con base en intereses y no en posiciones; con el aporte de propuestas objetivas y pensando en una relación para beneficio mutuo y a largo plazo.

Declaración de conflicto de interés: El autor no manifiesta conflictos de interés institucionales ni personales.

\section{Referencias}

Ansoff, H. I. (1957). Strategies for diversification. Harvard Business Review, 35(5), 113-124.

Biblioteca Virtual Universal. (2003). Tzu Sun: el arte de la Guerra. https://biblioteca.org.ar/libros/656228.pdf

Chandler, A. (1962). Strategy and Structure: Chapters in the History of the Industrial Enterprise. Massachusetts Institute of Technology Press.

Covey, S. (2003). Los 7 hábitos de la gente altamente efectiva: la revolución ética en la vida cotidiana y en la empresa. Paidós Plural.

Chase, R., Jacobs, F., \& Aquilano, N. (2009). Administración de operaciones: producción y cadena de suministros (12. ${ }^{\mathrm{a}}$ ed.). McGraw Hill.

Contreras, E. (2013). El concepto de estrategia como fundamento de la planeación estratégica. Pensamiento \& Gestión, (35), 152-181. https://www.redalyc.org/ pdf/646/64629832007.pdf

Cardillo, R., \& Szafranski, R. (2015). Tiro directo: ¿Ha muerto la estrategia? Air \& Space Power Journal, 27(4), 81-86.
https://www.airuniversity.af.edu/Portals/10/ASPJ_Spa nish/Journals/Volume-27_Issue-4/2015_4_11_cardi llo_s.pdf

Cronin, T. (2015). Aprender y pensar sobre liderazgo. Air \& Space Power Journal, 27(3), 36-50. https://www.airuni versity.af.edu/Portals/10/ASPJ_Spanish/Journals/Volu me-27_Issue-3/2015_3_06_cronin_s.pdf

Cambridge University. (s. f.). Negociación. En Cambridge Dictionary. https://dictionary.cambridge.org/es/ diccionario/ingles-espanol/negotiation

Fisher, R., \& Ury, W. (1985). ¡Sí, de acuerdo! Cómo negociar sin ceder. Editorial Norma.

Fernández-Osorio, A. E., Cufiño-Gutiérrez, F. N., Gómez-Diaz, C. A., \& Tovar-Cabrera, G. A. (2019). Dynamics of State Modernization in Colombia: The Virtuous Cycle of Military Transformation. Democracy and Security, 15(1), 75-104. https://www.academia.edu/44311150/Dyna mics_of_State_Modernization_in_Colombia_The_Vir tuous_Cycle_of_Military_Transformation

Galvis, R. A. (2006). Derecho Internacional Contemporáneo. Lo público, lo privado, los derechos humanos. Universidad del Rosario.

Hernández, R., Fernández, C., \& Baptista, M. (2014). Metodología de la investigación. McGraw Hill.

Harvard University. (2020). Negotiation Skills: Negotiation Strategies and Negotiation Techniques to help you become a better negotiator. https://professional.dce.harvard.edu/ negotiation-programs/?gclid=CjwKCAjw8KmLBhB8Ei wAQbqNoAGqvy9dL0uY9Dvane0R7ESAmWG84xFIM4D 5CahS-BJ7bJlIWsp6ahoCMm4QAvD_BwE

Jones, G., \& George, J. (2009). Administración contemporánea (6. ${ }^{a}$ ed.). McGraw Hill.

Kenichi, O. (1982). The Mind of Strategist. McGraw Hill.

Mintzberg, H. (1989). Mintzberg y la dirección. Ediciones Díaz de Santos.

Ogliastri, E. (2001). ¿Cómo negocian los colombianos? Alfaomega Cambio.

Organización para la Cooperación y el Desarrollo Económico \& Oficina Europea de Estadística. (2005). Manual de Oslo: guía para la recogida e interpretación de datos sobre innovación. (3. ${ }^{\mathrm{a}}$ ed.). OCDE; Eurostat. http://www.itq.edu. $\mathrm{mx} /$ convocatorias/manualdeoslo.pdf

Organización Mundial de la Propiedad Intelectual [OMPI]. (2019). La geografía de la innovación: núcleos locales, redes mundiales: informe mundial sobre la propiedad intelectual 2019. https://www.wipo.int/edocs/pubdocs/es/ wipo_pub_944_2019.pdf 
Paz, J. (2004). Negociación: competencia gerencial por antonomasia. Revista EIA, (1), 81-98. https://www.redalyc. org/pdf/1492/149217763008.pdf

Pulgarín, S., \& Rivera, H. (2012). Las herramientas estratégicas: un apoyo al proceso de toma de decisiones gerenciales. Criterio Libre, 10(16), 89-114. https://www.researchga te.net/publication/241754492_Las_herramientas_estrategi cas_un_apoyo_al_proceso_de_toma_de_decisiones_ gerenciales

Peña Chivatá, C., \& Díaz, D. (2019). El poder y la estrategia militar para la negociación e implementación de los acuerdos de paz en Colombia. Escuela Superior de Guerra. https://esdeguelibros.edu.co/index.php/editorial/cata $\log /$ view/39/42/681

Real Academia Española. (2019). Negociación. En Diccionario de la lengua española. https://dle.rae.es/?w=nego ciaci\%C3\%B3n

Serna, H. (2008). Gerencia estratégica: teoría-metodología, alineamiento, implementación y mapas estratégicos, índices de gestión (10. ${ }^{\mathrm{a}}$ ed.). 3R Editores.

Sarkis, B., \& Ocampo, J. (2015). Negociando con otras culturas: cómo negocian los colombianos según empresarios mexicanos. Civilizar, 15(29), 135-148. http://www.scielo. org.co/pdf/ccso/v15n29/v15n29a09.pdf
Schmitt, A., Raisch, S., \& Volberda, H. (2016). Strategic Renewal: Past Research, Theoretical Tensions and Future Challenges. International Journal of Management Reviews, 20(1), 1-18. https://www.researchgate.net/ publication/307922540_Strategic_Renewal_Past_Re search_Theoretical_Tensions_and_Future_Challenges_ Strategic_Renewal

Shonk, K. (2019). What is Negotiation? Harvard Law School. https://www.pon.harvard.edu/daily/negotiation-skillsdaily/what-is-negotiation/

Segura, C. (2020). El proceso de un estratega: menos directivos y más estrategas. KUMIAY Internacional.

Segura, C. (2021). Innovación de la estrategia militar: innovando con el apoyo de la herramienta moderna: Fortalezas, Oportunidades, Debilidades y Amenazas (FODA) + 1. Journal of the Americas, 3(2), 13-23. https://www. airuniversity.af.edu/Portals/10/JOTA/Journals/Volume\%203\%20lssue\%202/4-Segura_s.pdf

Villacis, D. (2014). La planificación estratégica como una herramienta de apoyo para determinar capacidades militares. Revista Política y Estrategia, (124), 13-36. https://www. politicayestrategia.cl/index.php/rpye/article/view/ $49 / 164$ 